\title{
Exploring the Use of Distributed Multiple Monitors within an Activity-Promoting Sit-and-Stand Office Workspace
}

\author{
Kathrin Probst ${ }^{1}$, David Lindlbauer ${ }^{1}$, Florian Perteneder ${ }^{1}$, Michael Haller ${ }^{1}$, \\ Bernhard Schwartz ${ }^{2}$, and Andreas Schrempf ${ }^{2}$ \\ ${ }^{1}$ Media Interaction Lab, University of Applied Sciences Upper Austria, Hagenberg/Austria \\ ${ }^{2}$ Medical Technology Department, University of Applied Sciences Upper Austria, Linz/Austria \\ mi-lab@fh-hagenberg . at
}

\begin{abstract}
Nowadays sedentary behaviors such as prolonged sitting have become a predominant element of our lives. Particularly in the office environment, many people spend the majority of their working day seated in front of a computer. In this paper, we investigate the adoption of a physically active work process within an activity-promoting office workspace design that is composed of a sitting and a standing workstation. Making use of multiple distributed monitors, this environment introduces diversity into the office workflow through the facilitation of transitions between different work-related tasks, workstations, and work postures. We conducted a background study to get a better understanding of how people are performing their daily work within this novel workspace. Our findings identify different work patterns and basic approaches for physical activity integration, which indicate a number of challenges for software design. Based on the results of the study, we provide design implications and highlight new directions in the field of HCI design to support seamless alternation between different postures while working in such an environment.
\end{abstract}

Keywords: Multi-Monitor, Distributed Display Environment, User Interaction.

\section{Introduction}

Over the past centuries, our lives have become more and more dominated by sedentary behaviors such as prolonged sitting. Particularly in the work context (e.g., office jobs), the number of tasks focused around sitting at a computer has increased markedly in the recent years [21, 22], although a growing body of research has connected this sedentary lifestyle to a dramatic increase of health-related risk factors (e.g., for cardiovascular or musculoskeletal diseases) [7, 30]. In contrast to that, a physically active work process promotes the avoidance of prolonged sedentary behavior and related degenerative phenomena. Thus, the World Health Organization (WHO) has identified the workplace as important area for setting health-promoting preventive measures with great potential to solve the problem of rapidly increasing health care costs [28]. 
Despite the growing evidence for the benefits of such workplace health promotion programs, interventions have proven to be limited though, as most of them pose demands on workforce commitment (e.g., gym classes), require employees to leave their desks for exercising, or are unlikely to be sufficient in the context of otherwise sedentary lifestyles [5]. Rather, the accumulation of small amounts of low-intensity physical activity throughout a day provides health benefits that may even exceed those associated with more vigorous activities [19]. Based on this insight, recent approaches have focused on the motivation of reduced sitting, increased walking or standing activities (e.g., taking breaks, postural diversity, stand-up meetings) [11], and the design of novel workspace environments that provide opportunities for integrating light activities into the daily office workflow [8, 18, 20, 23].

Since computer technologies have become an integral part of today's office work, the integration of physical activity into the work routine however brings along a number of challenges that have been rarely addressed by the HCI community so far [23]. The facilitation of a physically active work process poses demands on user interface technology (e.g., ergonomics) and software (e.g., interface design, interaction metaphors, data management) that may be quite different from those addressed in the design of today's stationary work environments. Addressing these demands while simultaneously ensuring work efficiency therefore needs to be considered for the design of future systems within increasingly flexible and mobile work environments.

In this paper, we study a sit-and-stand workspace environment that is designed to promote physical activity in a computer workplace (see Fig.1), which is composed of a traditional desk workstation and a standing table workstation facilitating execution of work-related tasks in sitting or standing posture [23]. It is not clear though, how users will use this novel workplace and what issues they need to overcome in order to work in a both efficient and physically active way. To get a better understanding of how people are working in such an environment and how this may imply requirements for the design of future computer systems, we performed an exploratory background study. In the remainder of this work, we present the observations of this study and the resulting implications on user behavior and HCI design.

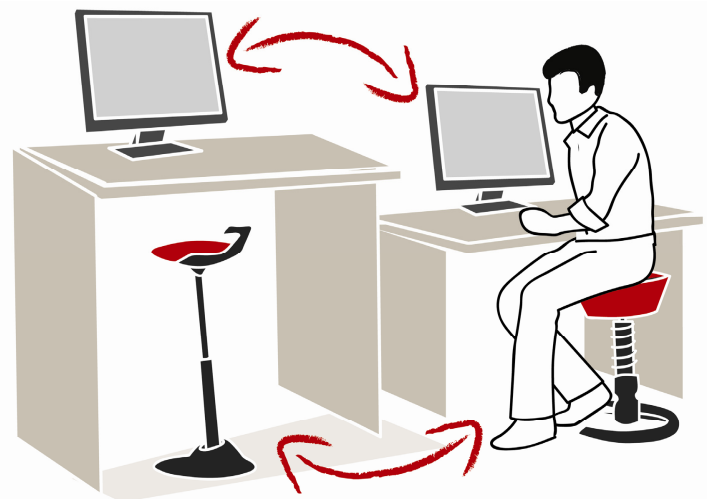

Fig. 1. A sit-and-stand workplace design integrating the concept of working in sitting and standing posture into the daily office workflow 


\section{Related Work}

\subsection{Workplace Health Promotion}

Different structural elements have been proposed for the promotion of physical activity in the workplace, such as standing desks [8], stepping devices [20], or "walk-andwork" workstations [18], which are directly integrated into the office environment. Especially sit-and-stand workstations have shown to offer high potential for effective workplace health intervention through variations between different postures. Previous studies showed a generally positive attitude of workers towards such workspaces [29], and significant reduction of musculoskeletal discomfort [12] without any negative effects on work performance [14]. While workers equipped with a sit-and-stand workspace tend to be positive about having the possibility to choose between sitting and standing, previous research also indicates that people sometimes tend to avoid standing posture because of ergonomic (e.g., workplace size), hardware-related (e.g., cable reach), or software-related (e.g., data access) constraints.

Another recent ergonomic approach for physical activity promotion [23] proposes a new concept of working "in-motion" and takes into account the smooth integration of hardware- and software-related structural elements to provide opportunities for office workers to seamlessly change between different work environments. Based on this work, we use a similar environment and extend it by equipping the design with a multi-monitor setup for further investigation.

\subsection{Multi-Monitor Usage}

Multi-monitor systems have been studied in a series of previous work. Initial evidence indicates that users can complete tasks more quickly with multiple monitors [6], but typically experience inefficiencies or frustrations (e.g., losing the cursor, bezel problems, distal information access problems, window management problems, task management problems, configuration problems [24, 26, 27]) that can be addressed by alterations to basic interaction [16]. Further, a number of observations of multimonitor users provide insights on the arrangement of digital information into focal and peripheral regions [9], window management [17] and other multi-monitor usage patterns. However, while much of the recent research involving multiple monitors has focused on compact setups where monitors are placed directly next to each other, a workplace design with two separate sitting and standing workstations introduces considerably larger distances than commonly used.

Since such environments have remained largely unconsidered by existing HCI research, we performed a background study to explore the usage in a real-world scenario, and provide insights into the user experience of working on a computer in sitting and standing posture. We analyze (1) the effects of such a distributed multiple monitor environment on user behavior, user interaction, usage patterns, and (2) how these may inform HCI design for supporting the concept of working while alternating between different work-related tasks, work postures, and corresponding workstations. 


\section{Concept and Method}

In accordance to a recently proposed activity-promoting workplace design [23], the basic concept of the present study involved providing participants with two workstations (see Fig.1) for execution of work-related tasks in sitting or standing posture, which consisted of two height-adjustable desks ${ }^{1}$ (Fig.2, left) and two active seats: a swopper ${ }^{2}$ active chair (Fig.2, middle) that supports dynamic sitting through $360^{\circ}$ tilting and vertical up-and-down flexibility, and a muvman chair ${ }^{3}$ (Fig.2, right) that can be used as a leaning chair to support working in standing posture.
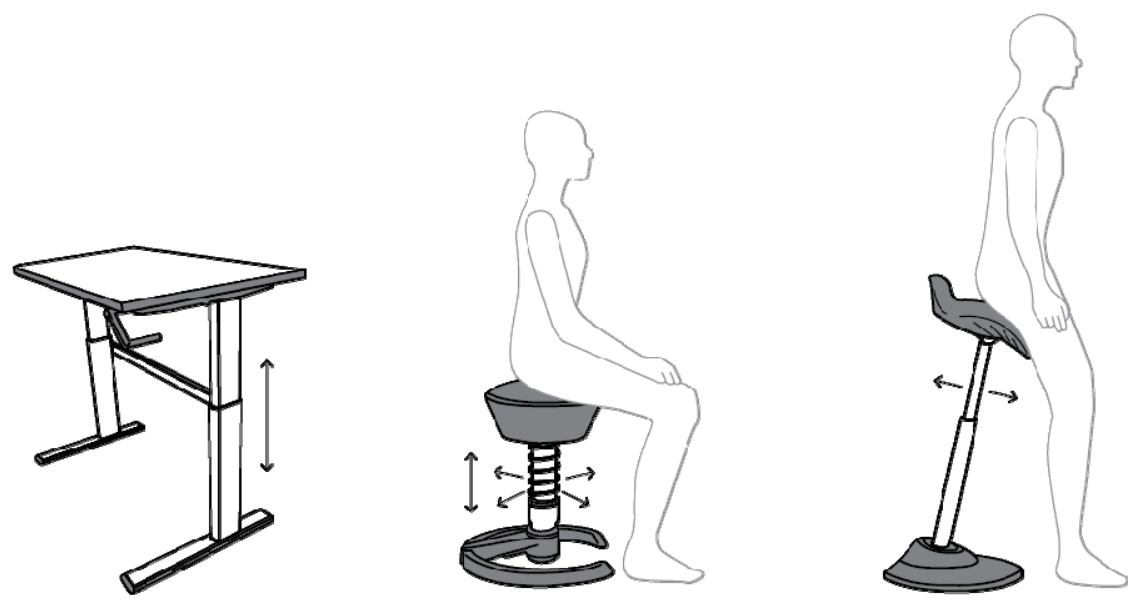

Fig. 2. As part of the study concept, participants were provided with two height-adjustable desks (left), a swopper active seat (middle), a muvman standing chair (right)

In the background study, we were interested in understanding several aspects of working within the proposed sit-and-stand workplace environment:

- How do participants organize the physical and hardware-related configuration of the workstations within the workspace, and how do people make use of the individual workstations within the environment (workspace configuration)?

- How do participants distribute their daily work time and work tasks among workstations within the workspace, and how is the usage influenced by the concept of working in different postures (workstation usage, switching behavior)?

- How well is this specific setup supported through current operating systems and software applications? Which problems and challenges arise (software support)?

\footnotetext{
${ }^{1}$ http: //actiforce.eu/nc/products/steelforce

${ }^{2}$ http: //www. swopper.de/en/swopper

${ }^{3}$ http: //www. swopper.de/en/muvman
} 


\subsection{Participants}

Five unpaid volunteers from the local university participated in a 4-week background study of their daily work activities within an activity-promoting workplace environment. All participants (4 male, 1 female; age 25-40) were experienced computer users, full-time employed as researchers in the field of computer science, ready to use such a setup to reduce sedentariness during the work routine. Four participants had no health problems, one participant reported on chronic back problems. All participants stated that they spent the majority of their work time (70-85\%) in front of the PC, and had used multi-monitor setups for six months to three years. They replaced their existing work environments with a provided activity-promoting workstation setup.

\subsection{Procedure and Setting}

At the beginning of the study, all participants were provided with identical basic office furniture sets as described in the previous section. Participants were instructed to arrange these components within their office space to form a personal sit-and-stand workspace environment that consists of a sitting and standing workstation (see Fig.1).

Participants were introduced to the adjustable features of the furniture and Ergonomics of Human System Interaction (ISO 9241). Corresponding to their individual work-related requirements, each workstation desk should be equipped with display and input devices connected to a single workplace computer to provide equal access from each workstation. Further, participants were introduced to their operating systems' native multi-monitor functionality ${ }^{4}$, and given free choice whether running their monitors in duplicated or extended mode throughout the course of the study.

\subsection{Data Collection and Analysis}

After setting up their personal workspace environments, participants' activities were observed for four subsequent weeks (20 work days). The individual workspaces were photographed, and specifications of the hardware equipment were collected. Participants reported on the time spent in sitting or standing posture, and overall work time at the end of each day. Throughout the study, six semi-structured interviews were conducted with every participant: after the first and third work day, and at the end of each work week. During the interviews, participants reported on their experience, and were assisted with suggestions for possible solutions to their reported problems. Interviews were audio-recorded (average duration 45 minutes) and affinity diagramming was used to analyze similarities, differences, and patterns in participants' behavior. In addition, a custom logging tool was installed on participants' computers, running in the background and collecting input and window events (similar to [17]). The data was used to gain insights on how often users switched between sitting and standing workstations, and which activities they performed in these locations.

\footnotetext{
4 The systems' multi-monitor settings could be accessed and changed by pressing $W i n+P$. http: //windows.microsoft.com/en-US/windows7 / Connect-to-a-projector
} 


\section{$4 \quad$ Results}

Based on the data collected during interviews and logging (on average 330,000 input events per day for each participant), we drew information on participants experiences, behavior and (computing) activities during the conducted study.

In this section, we present our results according to aspects that we were primarily interested in: how did participants combine the provided furniture components with hardware-related structural elements (workstation configuration), how did they integrate the concept of working in sitting and standing posture into their work routine (workstation usage, switching behavior), and to what extent this concept is supported by current software systems (software support).

\subsection{Workspace Configuration}

For all five participants, the arrangement of the provided furniture components resulted in a similar sit-and-stand workspace (see Fig.3). The tables were adjusted to participants' body height and arranged adjacent to each other, with a small gap of 10$15 \mathrm{~cm}$-either at a slightly tilted angle of approximately $45^{\circ}$ (four participants) or in straight line (one participant)-and were combined with the provided active seats to form the proposed sitting and standing workstations.
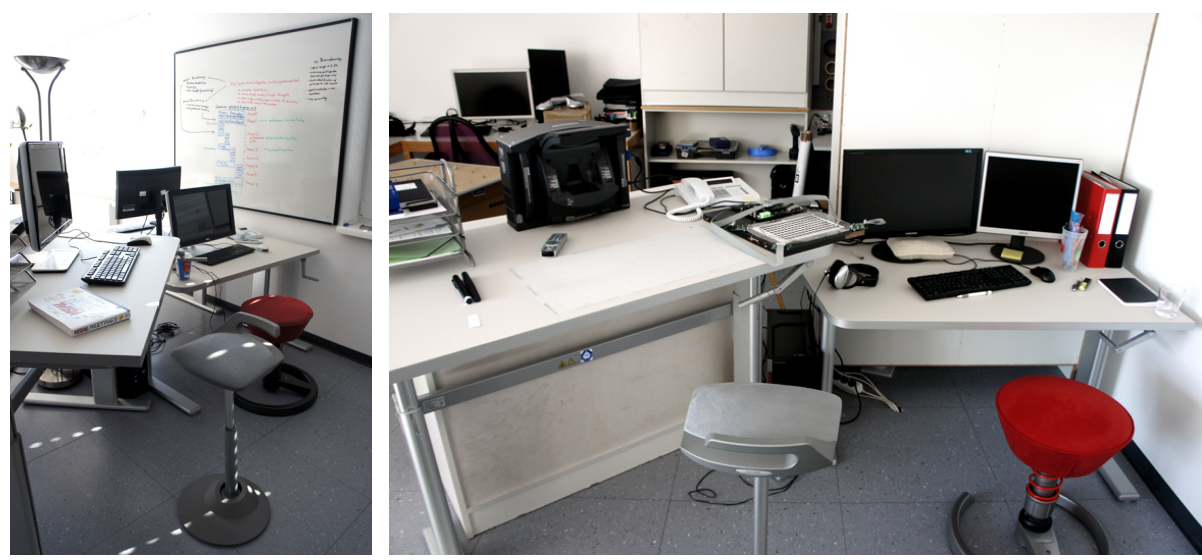

Fig. 3. Two participants' arrangements of their personal sit-and-stand workspace

Given the free choice how to combine the provided furniture with hardware components, participants further chose a similar configuration consisting of one computer (placed in a central position on the floor) with multiple connected monitors that were distributed across the tables. Four participants arranged a single monitor, keyboard, and mouse on each table (e.g., Fig.3, left). One participant had a dual-monitor setup on one table and a front-projected tabletop-display with stylus input on the other (Fig.3, right). All computers ran Microsoft Windows operating system (one with Windows 8, three with Windows 7, and one with Windows XP). 


\subsection{Workstation Usage}

During the study period, participants reported an average daily work time of 7.4 hours $(S D=1.86)$, of which they spent the major part of 6.0 hours $(S D=1.97)$ within their personal office workspace. During that time, their main work tasks (Fig.4, left) included software development, reading/writing (e.g., reports, articles, presentations), research/browsing, data analysis (e.g., statistics), communication (e.g., e-mail, telephone), meetings, and other (e.g., design, graphics, measurement) activities.
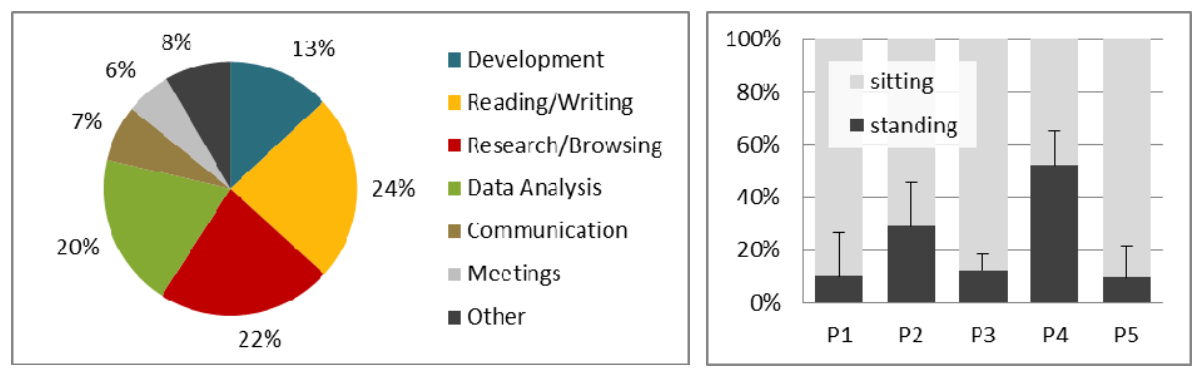

Fig. 4. Participants' reported daily work tasks (left) and sitting/standing times (right)

The average distribution of the reported sitting and standing times shows that all five participants made use of the opportunity to incorporate the concept of working in different postures into their regular office workflow. They reported spending on average $22.5 \%$ ( $S D=21.1 \%$ ) of their daily office time in standing posture (Fig.4, right). Given the relatively high variations, the participants' comments during the interviews provide further insights on a spectrum of different approaches for utilizing the novel sit-and-stand workspace. In-depth reports on the different aspects contributing to the above reported distribution are discussed in detail in the remainder of this chapter.

\subsection{Participants' Overall Experience}

To provide a brief overview of participants' overall experience, we present their basic approaches, behaviors and usage patterns over the course of the study-along with the daily sitting and standing times (average indicated through a horizontal line) that are depicted for each participant individually.

P1 used two monitors in extended mode, spent most of his work time using the primary screen at the sitting workstation, and switched to standing posture at rare intervalsvirtually treating it as an independent unit that provided him with a scope for handling specific work tasks in isola-

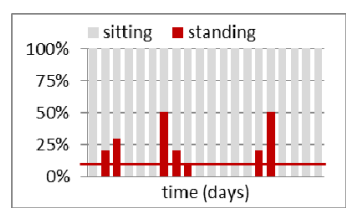
tion. Along with that, nevertheless, he experienced limitations during cross-monitor interaction (e.g., losing the mouse cursor during drag interactions) and during window switching (e.g., no taskbar on the secondary screen)-which did not encourage him to make use of the standing workstation on a more regular basis. 
$\mathbf{P 2}$ used two monitors in extended mode, mainly working at the primary screen at the sitting workstation, and switching to the standing workstation multiple times throughout a day to break up sitting periods from time to time. By doing so, however, he experienced a range of problems with in-

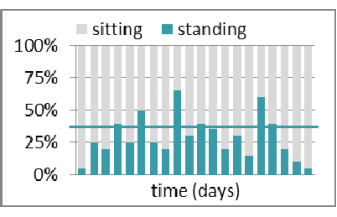
consistent window placement, during cross-monitor interaction (e.g., effort of dragging windows), and during window switching (e.g., no taskbar on the secondary screen). Therefore, after the second week of the study, he started to use the operating systems' native multi-monitor support-which enabled him to relocate the primary screen during workstation switches, and dissolved most previously existing problems.

P3 used two monitors in duplicated mode, spent most time at the sitting workstation, and tried to develop a routine to integrate working in standing posture into his workflow. After initially taking the opportunity to perform short email or browsing tasks at the standing workstation, he finally took a rather opportunistic approach of occasionally

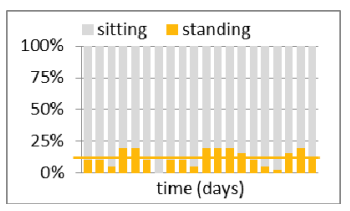
alternating his work posture at logical points within a task. He appreciated the convenience of switching between the duplicated monitors without effort, but raised concerns on peripheral distraction and privacy. To avoid these problems, he sometimes used the native multi-monitor support, but considered this as additional effort to some extent.

P4 used two monitors in duplicated mode, spent approximately equal amounts of his work time in sitting and standing posture, and alternated his work posture on a regular basis throughout a day. Having chronic back problems, he felt that this was a possibility to relieve the pressure on the spine, and appreciated the flexibility to switch between the

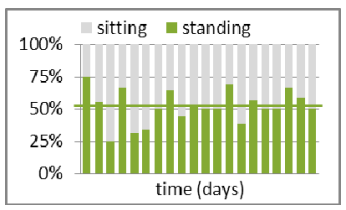
duplicated monitors whenever he wanted. Over the course of the study, some distinctive patterns started to evolve from his day-to-day work, as he tended to start the day with checking e-mails in standing posture, to work in sitting posture after lunch break, and to perform certain work tasks at one of the workstations (e.g., collaborative settings in standing posture, cognitive demanding tasks in sitting posture).

P5 used two monitors and a tabletop projection in extended mode, using the dual-monitor setup at the sitting workstation and the tabletop-setup at the standing workstation for debugging of pen-based input. During programming, he found that this worked well for him, as he perceived the

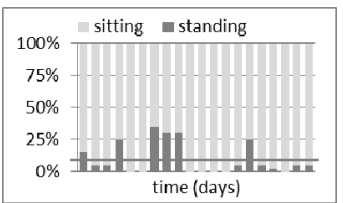
high-frequent regular switches as a welcome change. Due to the distinct separation of coding and debugging views and the pen input, he did not have to take any additional effort for dragging windows or moving the mouse cursor. Over time, however, he did not have any opportunity to work in standing posture besides of this specific implementation task and ended up spending the majority of the time seated. 


\subsection{Switching Behavior}

During a work day, the logged data shows that participants switched on average 6.27 times $(S D=8.47)$ between the sitting and standing workstations at intervals of 1.13 hours $(S D=1.12)$, with an average duration of 1.82 hours $(S D=1.88)$ in sitting and 0.44 hours $(S D=0.36)$ in standing posture. Comments from the interviews provide evidence for a variety of factors related to participants' physical and emotional personal condition, subject and progress of the current work task, or hardware- and software-related workspace configuration that were perceived to influence participants' switching behavior in a positive (encouraging) or negative (limiting) way. These reported influencing factors are discussed in detail in the remainder of this section and summarized in Table 1.

Table 1. Reported influencing factors with encouraging (+) or limiting (-) effects

\begin{tabular}{|c|c|c|c|c|c|c|}
\hline Category & Influencing Factor & $P 1$ & $P 2$ & $P 3$ & $P 4$ & $P 5$ \\
\hline \multirow{3}{*}{$\begin{array}{l}\text { Personal } \\
\text { Condition }\end{array}$} & Physical Discomfort & + & + & + & + & + \\
\hline & Personal Habits & - & & - & + & \\
\hline & Emotional Stress & & - & - & & \\
\hline \multirow{5}{*}{ Work Task } & New/Other Task & + & + & & + & + \\
\hline & Workstation Association & + & + & & & + \\
\hline & Logical Breakpoint & & + & + & + & \\
\hline & Cognitive Demand & & - & - & - & \\
\hline & Task Involvement & - & & - & & \\
\hline \multirow{4}{*}{$\begin{array}{l}\text { Workspace } \\
\text { Configuration }\end{array}$} & Collaboration & + & + & + & + & + \\
\hline & Accessibility & & + & + & + & \\
\hline & Physical Constraints & & & - & - & - \\
\hline & Software Limitations & - & - & - & - & - \\
\hline
\end{tabular}

Personal Condition. One consistent finding applicable for all participants is that physical discomfort of prolonged sitting (e.g., back muscle tension) or standing (e.g., fatigue, aching feet) was the main trigger to switch from one workstation to another. Participants stated to appreciate the possibility to bring diversity into their daily office routine by alternating between sitting and standing posture. This was especially true for $\mathrm{P} 4$, who had chronic spinal problems and he felt that regular posture changes relieved his back pain fundamentally. Besides of that, four participants (P1-P4) noted their switching behavior being dependent on personal habits to a certain extent. P4 for example, started nearly every work day in standing posture as he was generally feeling energized in the morning, and P1/P3 stated to rarely work in standing posture as they were not used to this kind of bodily load. Two participants (P2, P3) reported limited number of postural switches (i.e., preference of sitting posture) in situations of high emotional stress when they were feeling anxious or impatient. 
Work Task. Besides the triggers related to participants' personal condition, most of them (P1-P4) also stated that the start of a new task or switching the current task was commonly associated with postural changes. To find additional opportunities for switching their workstations on a frequent basis, two participants (P3, P5) further started to introduce direct association of workstations with particular tasks. P3 for example, was initially taking occasional breaks from his mainly seated day by performing short e-mail or browsing tasks at the standing workstation. P5 used the dualmonitor setup on the sitting workstation for his common work, and the projector-setup on the standing workstation for debugging activities. Along with that, however, they oftentimes ran into situations when their work tasks were not diverse enough to support a regular switching behavior. Given that, P5 tried to find additional tasks that he considered suitable to be performed at the standing workstation (e.g., reading on printed paper), while P3 changed his usage pattern after the first week of the study, thenceforth (similar to P2 and P4) rather changing his posture at logical breakpoints in-between the current task (e.g., at the end of a paragraph in a text). Limitations for switching behavior, on the other hand, were indicated by three participants (P2-P4) tending to avoid standing posture for tasks with relatively high cognitive demand (e.g., programming), and two participants (P1, P3) showing deep task involvement that made them simply "forget" about changing their posture when they were completely focused on the current task.

Workspace Configuration. Another interesting finding reveals that all participants pointed out the suitability of the standing workstation for collaborative activities. For typical settings in small groups of 2-4 people (e.g., discussions, showcasing), participants highlighted the affordances of the standing posture such as equal sight on the display surface and being at equal eye level. Closely related to that, the accessibility of the standing desk encouraged most participants (P2-P4) to use it as a first entrypoint after leaving the workspace temporarily for external activities (e.g., meetings, breaks). On the other hand, some participants (P3-P5) noted situations where physical constraints (e.g., hardware components, notes on printed paper) turned out as limitations to their switching behavior, and all participants consistently reported that software limitations (e.g. relocation of on-screen content) had a considerable effect on their switching behavior-which is discussed in detail within the following section.

\subsection{Software Support}

The analysis of the collected logging data shows that participants actively used their workplace PCs (incoming mouse, keyboard, or pen input events) on average 4.98 hours per day. Throughout a work day, participants used 5-12 different software programs (Fig.5, left), which included programming, text editing/viewing (e.g., text processor, PDF viewer), web browser, spreadsheet, communication (e.g., e-mail client, instant messenger), media (e.g., graphics/video/audio editor), and other (e.g., file browser) applications. 

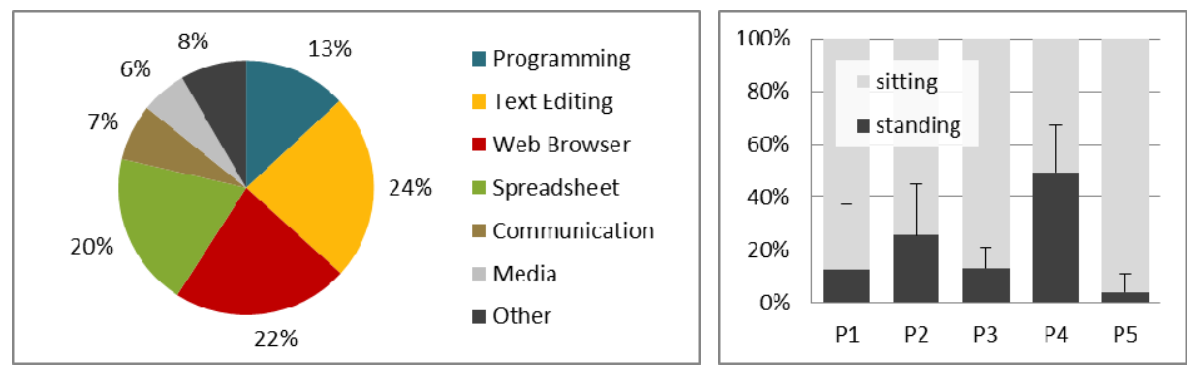

Fig. 5. Participants' logged software usage (left) and sitting/standing times (right)

The reported sitting/standing times (see Fig.4, right) are largely confirmed by the logging data (Fig.5, right), showing on average $20.61 \%(S D=21.42 \%)$ of the input time being recorded at the standing workstation. In addition to participants' different motivations for workstation switching, the distribution of monitors within the workspace had a major effect on their behavior. With a relatively large gap $(80-150 \mathrm{~cm})$ between monitors spread across the sitting and standing tables, all participants reported to perceive the workstations more or less as two separate units-feeling that they could use either the one or the other, but not both at the same time. As a result, two participants (P3, P4) decided to run their monitors in duplicated display mode, while the other three (P1, P2, P5) ran their setup in extended mode, and two participants (P2, P3) changed to a hybrid relocated display mode in the course of the study by manually switching the display configuration. Participants' experienced software support issues are discussed in the remainder of this section and summarized in Table 2.

Table 2. Reported software support issues and their occurrences per participant

\begin{tabular}{lccccc}
\hline Issue & $P 1$ & $P 2$ & $P 3$ & $P 4$ & $P 5$ \\
\hline Primary/Secondary Screen Distinction & $\bullet$ & $\bullet$ & & & $\bullet$ \\
Window Placement Inconsistency & $\bullet$ & $\bullet$ & & & $\bullet$ \\
Cross-Monitor Interaction & $\bullet$ & $\bullet$ & & & \\
Content Relocation Effort & $\bullet$ & $\bullet$ & $\bullet$ & & $\bullet$ \\
Peripheral Distraction & & & $\bullet$ & $\bullet$ & \\
Privacy & & & $\bullet$ & & \\
Waste of Resources & $\bullet$ & $\bullet$ & $\bullet$ & $\bullet$ \\
\hline
\end{tabular}

Extended Screen. In contrast to regular multi-monitor setups, the display units in our participants' setups were non-adjacent [26]. Consequently, none of them exhibited patterns like using the display space as a whole (e.g., straddling a window across monitors), using the two monitors in direct support of each other (e.g., reading on one screen while writing a text on the other), or for displaying peripheral information (e.g., at-a-glance e-mail notifications) [9]. Instead, participants used the two screens in a rather separate fashion, thereby experiencing a variety of problems that had a major influence on their usage of the single workstations. 
While the inherent primary/secondary screen distinction makes sense in a traditional extended desktop setting, this multi-monitor usage paradigm did not hold for the present setup. Two of the three extended screen users (P1, P2) reported window management limitations due to the taskbar, start menu, and task switching overview being displayed on the primary screen only. Consequently, while working on the secondary screen, they experienced difficulties to manage multiple windows without the visual feedback and one-click access functionality of these interface elements. As a result, P2 started to prepare well-defined work packages that did not require extensive window switching activities before switching to the secondary monitor at the standing workstation. P5 in contrast, reported to benefit from recently added multi-monitor support in the latest Windows version (Windows 8) providing him with the possibility to put a separate taskbar on each individual screen. A problem that however applied to all three extended screen users was the missing visual feedback of the quick-access task switcher $\left(\mathrm{Alt}+\mathrm{Tab}^{5}\right)$ being located on the primary screen by default.

Another issue reported by all extended screen users was the problem of window placement inconsistency. Most applications' inherent memory mechanisms [15] to open application windows on their last known location were holding only as long as people kept running a program at the same workstation (e.g., P5). Whenever participants were transitioning between workstations though, windows that continued to appear on the same (but not necessarily current) screen had a rather detrimental effect. $\mathrm{P} 1 / \mathrm{P} 2$ for example, reported that applications were frequently launched on the screen that they were currently not working at, that pop-up windows (e.g., notifications, file dialogs) easily went unnoticed on the second screen, and P5 experienced similar problems whenever he performed debugging tasks while sitting in some exceptional cases.

Associated with the above mentioned issues, participants oftentimes ran into situations when they had to apply cross-monitor interactions to shift digital content (e.g., windows, folders, icons) from one screen to another - thereby traversing the distance between the monitors by moving the mouse at one workstation to reach to a screen area on the other. Since the physical distribution introduced visual discontinuities [27], two participants (P1, P2) reported difficulties in navigating the mouse cursor, or reading text on the distant screen. Losing the mouse cursor [24] was reported to frequently occur in situations when the cursor was "left behind" during workstation switches, or when "disappearing" out of sight along the screens edges.

Finally, the need to associate changes in posture with interactions for digital content relocation was considered as a major limitation. Two participants (P1, P2) stated that they perceived it as annoying to bring windows from one side to the other during workstation switches. For P3 and P4, this additional effort was one main reason why they had decided to run the monitors in their workspace in duplicated display mode: "It's not the same as having two monitors on one desk. If the other monitor is far away and you're trying to see when you're trying to pass a window from one screen to the other, this is not so easy to do. (...) That's why I couldn't imagine working with the monitors in extended mode." (P3).

${ }^{5}$ http: //support.microsoft.com/kb/79869 
Duplicated Screen. Participants who used the setup in duplicated display mode (P3, P4) did not experience the same problems as extended screen users. By virtually having a single screen replicated on the extended monitor, they did not have to deal with discrepancies between primary and secondary screen, losing the mouse cursor, or the effort of moving digital objects from one screen to another. Having the exactly same view on all monitors, they were flexible to switch between the sitting and standing workstations at any desired point during task execution. Apart from that, duplicated screen users experienced other problems, though.

Having screen contents duplicated between workstation, participants reported feeling slight distraction due to on-screen interactions (e.g., mouse cursor movement, window pop-up) being visible on the second monitor within their peripheral vision. Closely related to this issue, one participant (P3) raised minor privacy concerns due to on-screen activities being observable for other people within the office space. While this was not a problem for P4, whose monitors were not visible from within the office space, the three extended screen users stated that privacy was a considerable reason why they had decided not to run their setups in duplicated mode.

A major concern however, was the waste of resources in terms of screen real estate and energy consumption, which was similarly indicated by the three extended screen users. By using only one of the two available monitors at a time, participants could not make use of a large part of their available screen space: "If I could work on both monitors at the same time, if I could control them in a better way... that would be much better. To be flexible to change between the two workstations, I should be able to make it feel more like one. (...) In terms of digital communication, it could be smoother, more continuous-instead of totally separated." (P1).

Relocated Screen. To avoid the disadvantages of both duplicated and extended display mode in the present multi-monitor setting, P2 and P3 started using the Windows native keyboard shortcut $\mathrm{Win}+\mathrm{P}$ for second screen projection after the first two weeks of the study. In this special usage scenario, postural changes were associated with pressing the keyboard shortcut to relocate the primary screen between sitting and standing workstation monitors-which resolved issues regarding primary/secondary screen, window placement, and cross-monitor interaction, and dissolved concerns about peripheral distraction, privacy, and energy consumption. Especially for P2, the new functionality changed his workstation switching behavior fundamentally, encouraging him to change his work posture at arbitrary points during a task. However, the major problem of losing a large part of available screen real estate remained unsolved.

Other remaining problems in this configuration were for example the monitor's latency to display content after enabling it with the shortcut. Further, it was not always clear which workstation would be enabled after pressing the keyboard shortcut, and which monitor would be turned on when participants returned to the workstation after prolonged inactivity (e.g., absence from the workspace due to external meetings). Furthermore, P2 felt like the black screen on the unused workstation was a minor barrier compared to an activated monitor, and P3 rarely made use of the screen relocation function because he considered this as additional effort to some extent. 


\section{$5 \quad$ Discussion and Implications}

Based on the described observations, we draw the following implications for the support of environments similar to the studied sit-and-stand workspace design.

\subsection{Basic Approaches for Physical Activity Integration}

From the findings of the study, we identify two basic approaches that were followed to incorporate the concept of working different postures into the daily office work:

- Task-Driven approaches are based on the concept of associating a work task with a specific workstation, thus implicitly motivating people to take up different postures throughout a work day. P5 for example, associated debugging activities with the standing workstation, P4 preferred the execution of collaborative tasks in standing posture, and P3 initially handled e-mail activities at the standing table.

- Self-Determined approaches are in contrast based on the concept of performing any work tasks at any workstation, leaving the participants free to change their posture at all times. P3 for example, proceeded to alternate between the sitting and standing posture every once in a while, or P4 always changed his work posture whenever he felt the desire to break continuous sitting or standing periods.

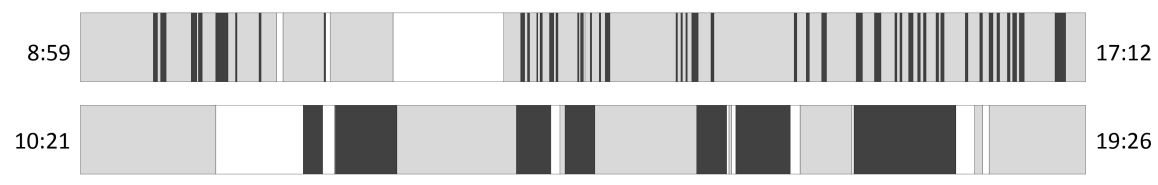

Fig. 6. Example distributions of computing events for task-driven (P5, top) and self-determined (P4, bottom) switching between sitting (gray) and standing (black) workstation.

Considering the experience of participants following a rather task-driven switching behavior, it becomes evident that this approach is strongly dependent on the daily work activities. While participants stated to perceive postural changes as rather natural side effect of their regular office work, it is on the other hand not suitable for tasks that are not performed on a regular basis. Self-determined switching behavior turned out as a flexible approach that leaves people free to carry out their work in any posture, but is on the other hand associated with a certain cognitive demand and might not work during phases of high involvement in the ongoing task. From the analysis of participants' switching patterns, we see that self-determined approaches resulted in significantly longer standing periods (Fig.6, bottom), while task-driven approaches resulted in a higher number of switches (Fig.6, top) during a work day.

Since both approaches have their pros and cons, and participants tended to not exhibit one of these two approaches exclusively, we think that it is essential to support a hybrid solution: while the task-driven approach has high potential to achieve an implicitly motivating effect, users should at the same time not be limited to exclusive association of task and workstation, but rather be able to switch in a self-determined manner whenever task-driven approaches are not applicable or undesirable. 
Furthermore, while our logging data confirmed findings on equal data-entry performance in any posture [14], participants in our study oftentimes preferred the sitting workstation, mainly due to reasons of personal habit or convenience. Especially for participants with self-determined switching behavior, we think that subtle reminding mechanisms (e.g., ambient displays [10]) can assist the adoption of a sustainable behavior change. For a task-driven approach, this may not be needed as long as the daily work routine consists of a diverse range of tasks. This is where assignment mechanisms (e.g., intelligent window arrangement algorithms [13]) can provide assistance for users to achieve a balanced task distribution among individual workstations.

\subsection{Supporting Distributed Multi-Monitor Usage}

Considering the study results, we see that physical separation introduced with distribution of multiple monitors across the work environment fundamentally changed the way people perceived and handled them. Although the monitors were connected to one machine, enlarged distances coupled with differences in height and relative orientation to the user introduced visual discontinuities [27], which presented major barriers to associated multi-monitor interaction. In contrast to adjacent multi-monitor setups, where users slightly rotate their neck to cover the increased screen space [26], the physical gaps in the present setup required them to additionally change their orientation or even physical location to switch focus between screen content spread across different monitors. To make effective use of both workstations, we identify a variety of challenges for designing future systems to support the physical distribution through exclusive, supportive, or peripheral multi-monitor usage [9]:

- Exclusive usage implies the association of the physical distribution with corresponding logical separation-virtually treating the monitor(s) and input devices at each workstation as a single logical unit. The basic functionality of the Win+P display switching mechanism could be extended or automated to activate a workstation monitor whenever the corresponding mouse or keyboard are operated.

Although exclusive monitor usage solves certain problems described earlier (e.g., window placement inconsistency, cross-monitor interaction), a major drawback remains. Participants expressed the desire to make use of the secondary monitor, since they felt like they were not using the full potential of their workstations. Concerns were raised that having two monitors but only having one in use was perceived as waste of resources (basically losing half of their working area).

- Supportive usage implies the association of the physical distribution with corresponding visual separation, thereby treating the displays at each workstation as visually isolated spaces. Digital barriers can serve as a basis for the restriction of mouse cursor movement [2], border snapping mechanisms, or consistent placement of windows on the current screen.

The effort of moving digital objects between monitors was identified as major barrier for fluent transitions from one workstation to another, implying the need to support effortless window management mechanisms such as grouping [25], switching, or relocating [1,3] digital content on the individual screens. 
- Peripheral usage implies the adaption of the user interface and interactions, since participants avoided interactions with the secondary monitor due to non-optimal mouse support and poor visibility of digital content across the distance in extended display mode. In response to that, interface elements on the distant screen could be enlarged and simplified to achieve a good readability, or convenient access to these elements should be supported through novel (multi-modal) interaction styles.

Essentially, users need to be able to equally access both workstations and the switching process needs to be effortless and seamless to maintain their motivation for the adoption of a physically active work process. Some participants were favoring the workstation with the primary screen (thus not switching workstations) because of existing software limitations. Avoiding these limitations to provide users with a seamless switching experience is a very important factor for this kind of activity-promoting office environments, though. Therefore, it seems crucial to gain knowledge about the user's activity (e.g., the currently used workstation) and properties of the surrounding environment (e.g., presence of other people). Based on that knowledge, an intelligent office space could for example respond to a user's activity by bringing digital objects into his focus of attention automatically [4].

\section{Conclusion and Future Work}

In this paper, we presented a background study with five office workers, who were carrying out their daily work tasks within a sit-and-stand workspace environment that follows the concept of integrating light activity into the daily work routine by providing the opportunity to work in sitting and standing posture [23]. Prior research in the field of multi-monitor usage is extended through the discussion of user behavior associated with the concept of working "in-motion". We indicated a number of challenges originating from user requirements within such an environment and discussed effects of different influencing factors on participants' workstation switching behavior. We identified two basic approaches for physical activity integration, and concluded that the situation-related support of both task-driven and self-determined patterns has high potential to provide users with a seamless switching experience. In a distributed multi-monitor setup, however, the physical separation of the individual displays has to be handled in a different way than in regular adjacent multi-monitor setups. Observations from our study showed that the distribution of monitors fundamentally changed the way people perceived and used them, and therefore experienced a variety of problems associated with running the monitors in extended, duplicated, or relocated mode. We highlighted resulting implications for the HCI design of future systems to support the seamless alternation of postures while working in such an environment.

Based on the findings of this study, future work will focus on the development of novel user interfaces and interaction techniques that provide an optimal interaction for exclusive, supportive and peripheral usage of distributed multi-monitor setups. Sensing methods will be developed on multiple levels to gain knowledge about the user's activity within the office workspace.

Another interesting insight provided by the presented study was that especially the standing workstation can be a great addition for collaboration and communication 
within an office space, which will also be addressed through the development of specific software support for such collaborative settings. Based on the findings on users' workplace switching behavior, we will examine these patterns more closely including the possibility to use and trigger them to promote workplace switching. Finally, future research will include additional studies with a larger number of participants and more diverse hardware setups (including e.g. notebooks and other mobile devices), and the investigation of challenges arising with the utilization of these setups within activity-promoting office environments.

Acknowledgements. We would like to thank Dr. Sheelagh Carpendale and the InnoVis group at the University of Calgary for their ideas and advice. Funding for this research was provided by the Austrian Research Promotion Agency GmbH (FFG) within the Bridge project \#2579917.

\section{References}

1. Baudisch, P., Cutrell, E., Robbins, D., Czerwinski, M., Tandler, P., Bederson, B., Zierlinger, A.: Drag-and-Pop and Drag-and-Pick: techniques for accessing remote screen content on touch- and pen-operated systems. In: Interact 2003, pp. 57-64. IOS Press, Amsterdam (2003)

2. Benko, H., Feiner, S.: Pointer Warping in Heterogeneous Multi-Monitor Environments. In: GI 2007, pp. 111-117. ACM Press, New York (2007)

3. Biehl, J., Bailey, B.: ARIS: An Interface for Application Relocation in an Interactive Space. In: GI 2004, pp. 107-116. Canadian Human-Computer Communications Society, London (2004)

4. Brumitt, B., Meyers, B., Krumm, J., Kern, A., Shafer, S.: EasyLiving: Technologies for Intelligent Environments. In: Thomas, P., Gellersen, H.-W. (eds.) HUC 2000. LNCS, vol. 1927, pp. 12-29. Springer, Heidelberg (2000)

5. Craft, L.L., Zderic, T., Gapstur, S., Vaniterson, E., Thomas, D., Siddique, J., Hamilton, M.: Evidence that women meeting physical activity guidelines do not sit less: An observational inclinometry study. International Journal of Behavioral Nutrition and Physical Activity $9,122(2012)$

6. Czerwinski, M., Smith, G., Regan, T., Meyers, B., Robertson, G., Starkweather, G.: Toward Characterizing the Productivity Benefits of Very Large Displays. In: Interact 2003, pp. 9-16. IOS Press, Amsterdam (2003)

7. Freak-Poli, R., Wolfe, R., Peeters, A.: Risk of Cardiovascular Disease and Diabetes in a Working Population With Sedentary Occupations. Journal of Occupational and Environmental Medicine 52(11), 1132-1137 (2010)

8. Gilson, N., Suppini, A., Ryde, G., Brown, H., Brown, W.: Does the use of standing "hot" desks change sedentary work time in an open plan office? Preventive Medicine 54(1), 65-67 (2012)

9. Grudin, J.: Partitioning Digital Worlds: Focal and Peripheral Awareness in Multiple Monitor Use. In: CHI 2001, pp. 458-465. ACM Press, New York (2001)

10. Haller, M., Richter, C., Brandl, P., Gross, S., Schossleitner, G., Schrempf, A., Nii, H., Sugimoto, M., Inami, M.: Finding the right way for interrupting people improving their sitting posture. In: Campos, P., Graham, N., Jorge, J., Nunes, N., Palanque, P., Winckler, M. (eds.) INTERACT 2011, Part II. LNCS, vol. 6947, pp. 1-17. Springer, Heidelberg (2011) 
11. Healy, G., Lawler, S., Thorp, A., Neuhaus, M., Robson, E., Owen, N., Dunstan, D.: Reducing prolonged sitting in the workplace. Human Factors and Ergonomics Society, Melbourne (2012)

12. Hedge, A., Ray, E.J.: Effects of an electric height-adjustable worksurface on self-assessed musculoskeletal discomfort and productivity in computer workers. In: HFES 2004, pp. 1091-1095. Mira Digital Publishing, Brentwood (2004)

13. Henderson, D., Card, S.: Rooms: The Use of Multiple Virtual Workspaces to Reduce Space Contention in a Window-Based Graphical User Interface. ACM Transactions on Graphics 5(3), 211-243 (1986)

14. Husemann, B., Von Mach, C., Borsotto, D., Zepf, K., Scharnbacher, J.: Comparisons of Musculoskeletal Complaints and Data Entry Between a Sitting and a Sit-Stand Workstation Paradigm. Human Factors 51(3), 310-320 (2009)

15. Hutchings, D., Stasko, J.: Consistency, Multiple Monitors, and Multiple Windows. In: CHI 2007, pp. 211-214. ACM Press, New York (2007)

16. Hutchings, D., Stasko, J., Czerwinski, M.: Distributed Display Environments. Interactions 23(6), 50-53 (2005)

17. Hutchings, D.R., Smith, G., Meyers, B., Czerwinski, M., Robertson, G.: Display Space Usage and Window Management Operation Comparisons between Single Monitor and Multiple Monitor Users. In: AVI 2004, pp. 32-39. ACM Press, New York (2004)

18. Levine, J., Miller, J.: The energy expenditure of using a "walk-and-work" desk for office workers with obesity. British Journal of Sports Medicine 41(9), 558-561 (2007)

19. Levine, J.A.: Non-Exercise Activity Thermogenesis (NEAT). Best Practice \& Research: Clinical Endocrinology \& Metabolism 14(4), 679-702 (2002)

20. McAlpine, D., Manohar, C., McCrady, S., Hensrud, D., Levine, J.: An office-place stepping device to promote workplace physical activity. British Journal of Sports Medicine 41(12), 903-907 (2007)

21. Miller, R., Brown, W.: Steps and sitting in a working population. International Journal of Behavioral Medicine 11(4), 219-224 (2004)

22. Owen, N., Bauman, A., Brown, W.: Too much sitting: a novel and important predictor of chronic disease risk? British Journal of Sports Medicine 43(2), 81-83 (2009)

23. Probst, K., Leitner, J., Perteneder, F., Haller, M., Schrempf, A., Glöckl, J.: Active Office: Towards an Activity-Promoting Office Workplace Design. In: CHI Ext. Abstracts 2012, pp. 2165-2170. ACM Press, New York (2012)

24. Robertson, G., Czerwinski, M., Baudisch, P., Meyers, B., Robbins, D., Smith, G., Tan, D.: The Large-Display User Experience. IEEE Comput. Graph. Appl. 25, 2-9 (2005)

25. Smith, G., Baudisch, P., Robertson, G., Czerwinski, M., Meyers, B., Robbins, D., Andrews, D.: GroupBar: The TaskBar Evolved. In: OZCHI 2003, pp. 34-43. CHISIG of Australia, Canberra (2003)

26. Swaminathan, K., Sato, S.: Interaction Design for Large Displays. Interactions 4(1), 15-24 (1997)

27. Tan, D., Czerwinski, M.: Effects of Visual Separation and Physical Discontinuities when Distributing Information across Multiple Displays. In: Interact 2003, pp. 252-255. IOS Press, Amsterdam (2003)

28. WHO: Preventing Noncommunicable Diseases in the Workplace through Diet and Physical Activity: WHO/World Economic Forum Report of a Joint Event (2008)

29. Wilks, S., Mortimer, M., Nylén, P.: The introduction of sit-stand worktables; aspects of attitudes, compliance and satisfaction. Applied Ergonomics 37(3), 359-365 (2006)

30. Wilmot, E., Edwardson, C., Achana, F., Davies, M., Gorely, T., Gray, L.J., Khunti, K., Yates, T., Biddle, S.: Sedentary time in adults and the association with diabetes, cardiovascular disease and death: systematic review and meta-analysis. Diabetologia 55(11), 2895-2905 (2012) 